\title{
Die Klassifizierung von Makrochromosomen in Vogelskaryotype mittels eines Indexes der relativen Länge
}

\author{
Tomáš Husák, Vladimír Srb und Bohumil Král \\ Medizinische Fakultät der Karlsuniversität in Königgrätz \\ Tchechoslowakei
}

Eingegangen am März 18. 1967

Schneller Wuchs der Kenntnisse über die Chromosome von verschiedensten Pflanzen. sowie Tierarten brachte das Aufkommen zahlreicher karyotypologischer Systeme sowie Terminologien hervor. Die humane Zytogenetik ging auf die einheitliche Chromosomeklassifizierung vor allem auf Grund der Konferenzen über den menschlichen Karyotypus in Denver (Denver Report 1960) und in London (London Report 1963) ein.-Um solche einheitliche Klassifizierung bemühen sich auch Pflanzenzytogenetiken (Battaglia 1955, 1964, Murin 1966). Bloß in Tierkaryotypologie herrscht immerwährend bestimmtes Vakuum.Insbesonders die Vogelkaryotypologie ist sehr mühsam (z.B. Gallus gallus f. domestica), weil der zahlreich vorkommende jedoch bis jetzt nicht genau festgelegte Karyotyp-2n>70zweierlei Chromosome umfaßt: 6 Paare Makrochromosome und veränderliche hohe Zahl von Mikrochromosomen (Newcomer 1957, 1959, Van Brink 1959, Frederic 1961, Ohno 1961, Ford und Woollam 1964, Owen 1965, Srb et al. 1966 u.a.)-siehe Abb. 1.

\section{Die Wahl eines zweckmäßigen Indexes}

Von zahlreichen Autoren machte Frédéric als einziger einen Versuch Indexe der Denvernomenklatur auf Chromosome bei Gallus gallus f. domestica zu applizieren. Infolge der schwankenden Zahl der schwer zufaßbaren Mikrochromosome benutzte er hauptsächlich so genannten zentromerischen Index. Sein weiterer Beitrag besteht im Entwurf eines Indexes der relativen Länge (indice de grandeur relative-I.G.R.): Die Länge eines jeden Chromosomes ist durch Verhältnis zur als 100\% charakterisierten Länge des längesten Chromosomes zum Ausdruck gebracht; mit anderen Worten: Index eines als $k$-bezeichneten Chromosomes $\left(I_{k}\right)$ ist seine Länge $\left(x_{k}\right)$ dividiert durch Länge eines längsten Chromosomes $\left(x_{1}\right.$, wobei $\left.x_{1}=100 \%\right)$, oder $I_{k}=x_{k} / x_{1}$. Man muß bemerken, daß der angeführte Index bloß die Makrochromosome betrifft.

Für mathematisch genauere Klassifizierung von Hühnermakrochromosomen wollen wir einen anderen Index des relativen Länge der Makrochromosomen, so genannten H.S.K. - Index, vorschlagen, dessen wörtliche Formel folgendermäßen lautet: Index eines als $k$-bezeichneten Chromosomes des betreffenden Karyotypes $\left(I_{k}\right)$ ist durch die Länge dieses $k$-Chromosomes $\left(x_{k}\right)$ dividiert durch Durchschnittslänge der Makrochromosome $(\bar{x})$ gegeben, oder $I_{k}=x_{k} / \bar{x}$. 
Es ist klar, daß die Relation der Indexe im Frédéricschen Systeme sowie im demselben von uns vorgeschlagenen gleichbleibend ist. Es dürfe aber die Frage erötert werden, wie sich in beiden Fällen der auf Grund eines konkreten jedoch nicht ganz genauen Messens festgestellte Index von dem wirklichen einem ideelen Messen entsprechenden Indexe abweichen wird. Die Ungenauigkeit in Bestimmung eines $k$-Chromosomes ist erstens durch Unvollkommenheit der Methodik (der Fehler bleibt ja beiden Indexen gleich), zweitens durch Ungenauigkeit des Wertes $\bar{x}$ im unseren Beispiele und des Wertes $x_{1}$ im Frédéric's Beispiele bedingt. Aus Gesetzmäßigkeiten der statistischen Mathematik geht jedoch hervor, daß der Wert $\bar{x}$, d.h. die Durchschnittslänge der Chromosome, durch wesentlich kleineren Fehler als der Wert $x_{1}$, d.h. die Länge eines längsten Chromosomes, belastet wird. Es ist nämlich bekannt, daß die maßgebende Abweichung des Durchschnittes $\left(\sigma_{\bar{x}}\right)$ bei $n$ Elementen $V \bar{n}$-mal kleiner ist, als dieselbe Abweichung der Veränderlichen. Man muß daher annehmen, der Index des Fréd'ricschen Systems dürfte mehr den Einflüssen der zufälligen Fehler und der Unregelmäßigkeiten als Index in unserem Klassifizierungssysteme unterworfen sein.

\section{Beglaubigung der Vorteilhaftigkeit des H.S.K.-Indexes}

Wir haben theoretisch deduziert, $\mathrm{da} \beta$ der von uns benutzter Index zweckmäßiger ist, weil dessen Grundlage (arithmetischer Durchschnitt) mehr konstante und charakteristische Parameter darstellt, als die Grundlage des Frédéricschen Systemes (die Länge eines längsten Chromosomes). Um diese Hypothese praktisch zuüberprüfen, wurden auf beide Weisen die Makrochromosome von vier durch direkte Bearbeitung eines Knochenmarkes unter Applikation des Kolchicines (Srb, Kràl, und Hroch 1967) gewonnenen Karyo-

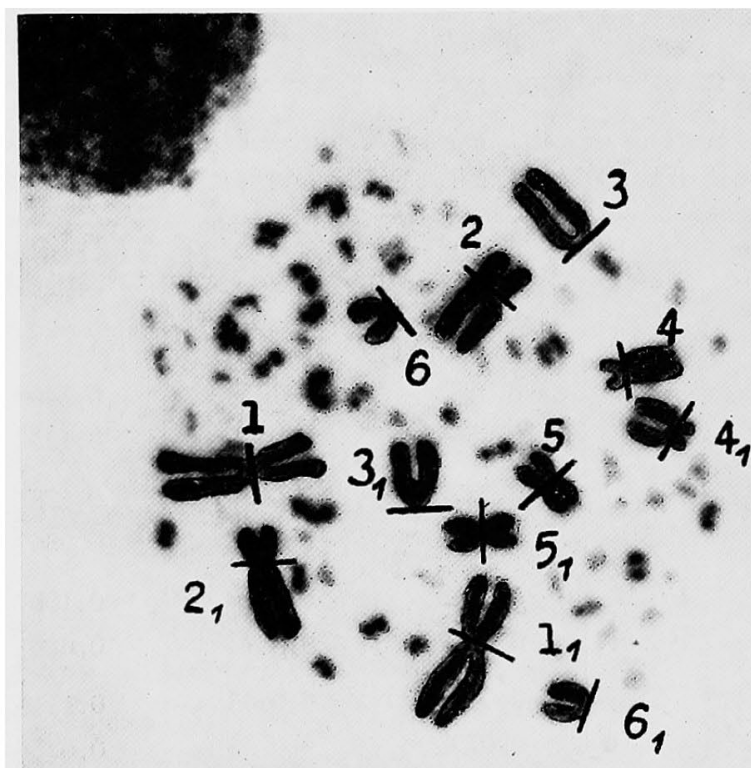
typen vielfältig durchgemessen. Man hat den Fehler in der Messung sowie dessen Einfluß auf beide Indexe verfolgt,

Abb. 1. Karyotyp von Gallus gallus f. domestica. 1 $1_{1} ; 2,2_{1} ; 3,3_{1}, 4,4_{1} ; 5,5_{1} ; 6,66_{1}$-Ein Paar von homologen Makrochromosomen (5, $5_{1}=Z Z$ Chromosome). Ein Streich über den Makrochromosomen bezeichnet die Lage der Zentromere. Photo VI. Srb. 
wie z.B. derselbe aus den Karyotyps Nr. 1 Gallus gallus f. domestica charakterisierenden Werten in Table 1 resultiert.

Die Ungenauigkeitsgrad (der Durchschnittfehler) bei Feststellung eines zahlmäßigen Wertes ist durch das Verhältnis zwischen der ausschlaggebender

Tab. 1. Mathematische Charakteristiken des Karyotypus $\mathrm{Nr}$. 1 von Gallus gallus f. demestica nach den Indizes L.G.R. (Frédéric-1961) und H.S.K. (Husák, Srb und Král 1966)

\begin{tabular}{|c|c|c|c|c|c|c|}
\hline $\begin{array}{l}\text { Zahl des } \\
\text { Makro- } \\
\text { chro- } \\
\text { mosoms }\end{array}$ & $\begin{array}{l}\text { Zahl der } \\
\text { Mes- } \\
\text { sungen } \\
(n)\end{array}$ & Durchschnitt & $\begin{array}{l}\text { Mitlerer } \\
\text { Fehler } \\
\text { (a) }\end{array}$ & $\begin{array}{c}\text { Mitlerer } \\
\text { Durchschnitts- } \\
\text { fehler } \\
\left(v_{x}\right)\end{array}$ & $\begin{array}{l}\text { Streuung } \\
\qquad\left(\sigma^{2}\right)\end{array}$ & $\begin{array}{c}\text { Durchschnitt- } \\
\text { streuung } \\
\left(\sigma_{\bar{x}}\right)^{2}\end{array}$ \\
\hline
\end{tabular}

I.G.R. Index (Frédéric 1961)

\begin{tabular}{l|l|l|l|l|l|l}
1 & 40 & 1,0000 & 0,0000 & 0,0000 & 0,0000 & 0,0000 \\
2 & 40 & 0,6837 & 0,0653 & 0,0103 & 0,0043 & 0,0001 \\
3 & 40 & 0,4534 & 0,0458 & 0,0072 & 0,0021 & 0,0001 \\
5 & 40 & 0,4978 & 0,0698 & 0,0110 & 0,0049 & 0,0001 \\
6 & 40 & 0,4189 & 0,0505 & 0,0080 & 0,0025 & 0,0001 \\
& 40 & 0,2582 & 0,0452 & 0,0072 & 0,0020 & 0,0001
\end{tabular}

H.S. Index (Husák, Srb und Král 1966)

\begin{tabular}{l|l|l|l|l|l|l}
1 & 40 & 1,8160 & 0,0915 & 0,0145 & 0,0084 & 0,0002 \\
2 & 40 & 1,2370 & 0,0816 & 0,0129 & 0,0067 & 0,0002 \\
3 & 40 & 0,8211 & 0,0733 & 0,0116 & 0,0054 & 0,0001 \\
4 & 30 & 0,9016 & 0,1128 & 0,0178 & 0,0127 & 0,0003 \\
5 & 40 & 0,7577 & 0,0744 & 0,0118 & 0,0055 & 0,0001 \\
6 & 40 & 0,4666 & 0,0698 & 0,0110 & 0,0049 & 0,0001
\end{tabular}

Tab. 2. Gegenüberstellung des Verhältnisses des mittleren Fehlers ( $\sigma$ ) zum Durchschnitt

$(\bar{x})$ bei den Indizes I.G.R. (Frédéric 1961) und H.S.K. Husák, Srb, und Král 1966).

\begin{tabular}{|c|c|c|c|c|c|c|}
\hline \multirow{2}{*}{$\begin{array}{c}\text { Zahl des } \\
\text { Karyo- } \\
\text { typus }\end{array}$} & \multirow{2}{*}{ Index } & \multicolumn{5}{|c|}{ Makrochromosom } \\
\hline & & 2 & 3 & 4 & 5 & 6 \\
\hline \multirow{2}{*}{1} & I.G.R & 0,0955 & 0,1010 & 0,1402 & 0,1205 & 0,1750 \\
\hline & H.S.K. & 0,0659 & 0,0892 & 0,1251 & 0,0981 & 0,1495 \\
\hline \multirow{2}{*}{2} & I.G.R. & 0,1338 & 0,0956 & 0,1283 & 0,1211 & 0,1698 \\
\hline & H.S.K. & 0,0833 & 0,0437 & 0,0867 & 0,1297 & 0,1266 \\
\hline \multirow[t]{2}{*}{3} & I.G.R. & 0,1463 & 0.1136 & 0,1007 & 0,1169 & 0,1092 \\
\hline & H.S.K. & 0,0837 & 0,0674 & 0,0581 & 0,0612 & 0,0575 \\
\hline \multirow{2}{*}{4} & I.G.R. & 0,0839 & 0,0906 & 0,1115 & 0,1412 & 0,0975 \\
\hline & H.S.K. & 0,0736 & 0,0568 & 0,0735 & 0,1029 & 0,1016 \\
\hline
\end{tabular}

Abweichung (=mitlerer Fehler, $\sigma)$ und dem Durchschnitte eines betreffenden Indexes $(\bar{x})$ als $\sigma / \bar{x})$ gegeben. Wir haben schon oben unser theoretisches Resultat angeführt der Durchschnittfehler bei Festsetzung eines H.S.K.Indexes dürfte kleiner sein, als derselbe bei Festsetzung des I.G.R.-Indexes. 
Hiebei gilt allgemeine Regel, daß je kleiner der zahlmäßige Wert des Verhältnisses der auschlaggebender Abweichung zum Durchschnitte ist, desto genauer wird der Indexwert festgestellt.

Die Vergleichung der Werte dieses Durchschnittfehlers, welche durch vierzigfaches Durchmessen von Hühnermakrochromosomen bei vier Karyotypen herausgefördert werden sind, ist in der $\mathrm{Tab} .2$ angeführt.-Aus denselben geht es hervor, daß der Durchschnittsfehler bei Indexfestsetzung im von uns vorgeschlagenen Systeme kleiner ist (mit Ausnahme von zwei Wertangaben), als im Frédéricschen Systeme. Hiemit haben wir unsere Schlußfolgerungen über den Vorzug des H.S.K.-Indexes auch experimental bestätigt.

Man muß hiezu noch bemerken, daß Frédéricscher I.G.R.-Index keine Information über die relative Länge eines längstens Chromosomes darbietet, indem derselbe apriorisch als Eins gewählt wird (Index 1,00), wogegen von uns vorgeschlagener H.S.K.-Index diese Information gewährt.

\section{Zusammenfassung}

Auf Grund einer mathematischen Analyse schlugen die Autoren vor und gleichzeitig beglaubigten im Falle Gallus gallus f. domestica einen neuen Index zur Bewertung von Vogelmakrochromosomen. Diesen Index einer relativen Länge der Makrochromosome, so genannten H.S.K.-Index $\left(I_{k}\right)$, charakterisieren als Längeverhältnis eines bestimmten Makrochromosomes $\left(x_{k}\right)$ zur Durchschnittslänge aller Makrochromosomes eines gegebenen Karyotypes $(\bar{x}): I_{k}=x_{k} / \bar{x}$.

Den ausgeführten Index beglichen mit dem Frédéricschen I.G.R.-Index und bewiesen, der H.S.K.-Index sei genauer und gewähre mehrere Informationen.

Zur Berechnung von mathematischen Charakteristiken beider Indexe wurde automatischer Zählapparat Odra 1003 benutzt.

\section{Summary}

Classification of macrochromosomes in avian karyotype using an index of relative length

On the basis of mathematical analysis the authors have prepared a new index of relative length of the macrochromosomes in the Gallus gallus $\mathrm{f}$. domestica, so-called H.S.K.-index $\left(I_{k}\right)$ which they characterize as the ratio of the length of certain $(k-)$ macrochromosome $\left(x_{k}\right)$ to the average length of all macrochromosomes of a given karyotype $(\bar{x}): I_{k}=x_{k} / \bar{x}$.

The above mentioned index was compared with Frédéric's index of the relative length of the macrochromosomes ("indice de grandéur relative", I.G.R.-index). After the analysis of the values gained by both procedures, they have suggest H.S.K.-index for more accurate one. 
For calculating mathematical characteristics of both indices they used automatic computer Odra 1003.

Die Danksagung: Unser aufrichtigen Dank gehört den Arbeitern des Berechnungs. centrum (V. Černý und K. Širůčková) bei medizinischen Fakultät der Karlsuniversität in Hradec Králové und der Frau M. Eib für die technische Mitarbeit.

\section{Literatur}

Battglia, R. 1955. Chromosome morphology and terminology. Caryologia 8: 179-187.

- 1964. Cytogenetics of B-chromosomes. Caryologia 17: 245-299.

Denver Report 1960. A proposed standard system of nomenclature of human mitotic chromosomes. Jour. of Hered. 51: 214-221.

Ford, E. H. R. and Woollam, D. H. M. 1964. Testicular chromosomes of Gallus domesticus. Chromosoma (Berlin) 15: 568-578.

Frédéric, J. 1961. Contribution à l' étude du caryotype chez le poulet. Arch. de Biol. 72: 185-209.

London Report 1963. The London conference on the normal human karyotype. Cytogenetics 2: 264-268.

Murín, A. 1966. Metodické poznámky k přípravě chromozomálného atlasu flory Slovenska. Bratislava, 9 stran.

Newcomer, E. H. 1967. The mitotic chromosomes of the domestic fowl. Jour. of Hered. 48: $227-234$.

- 1959. The meiotic chromosomes of the fowl. Cytologia 24: 403-410.

Ohno, S. 1961. Sex chromosomes and microchromosomes of Gallus domesticus. Chromosoma (Berlin) 11: 484-498.

Owen, J. J. T. 1965. Karyotype studies on Gallus domesticus. Chromosoma (Berlin) 16: 601-608.

Srb, V., Král, B. a Hroch, M. 1966. Préparation des lames de la médulle et des cultures tissulaires pour l'analyse chromosomale à Gallus gallus f. domestica. C. R. Soc. biol. Paris, $160 / 10 /$ : 1983-1986.

Van Brink, J. M. 1959. L'expression morphologique de la digamétic chez les Sauropsidés et les Monotrémes. Chromosoma (Berlin) 10: 1-72. 